\title{
Costs and Utilization of Triptan Users Who Receive Drug Prophylaxis for Migraine Versus Triptan Users Who Do Not Receive Drug Prophylaxis
}

\author{
LIDA R. ETEMAD, PharmD, MS; WINNIE YANG, PharmD, BCPS; DENISE GLOBE, PhD; \\ ARIE BARLEV, PharmD, MS; and KATHLEEN A. JOHNSON, PharmD, PhD
}

\begin{abstract}
OBJECTIVES: The objectives were 2-fold: (1) to describe the utilization patterns of new users of triptan therapy and (2) to measure the direct (pharmacy and medical) costs of migraine-related health care services in moderate-to-severe migraine patients treated with drug prophylaxis compared with migraine patients who are not treated with drug prophylaxis.

METHODS: A retrospective administrative database study was conducted from the perspective of a managed care health plan. Patients initiating triptan therapy were identified, and utilization in the 12 months following initiation of drug therapy was determined. In addition, moderate-to-severe migraine patients were identified based on the quantity of triptan medication dispensed. Patients were classified as utilizing or not utilizing migraine prophylaxis. Migraine-specific health services costs in the 12 months following identification were determined. A multivariate ordinary least squares regression model was constructed to determine the impact of the use of drug prophylaxis on total cost. Utilizing the model, the difference in health services costs was predicted for each subject and the average treatment effect was computed.
\end{abstract}

RESULTS: Thirty-nine percent of new triptan users received only 1 triptan claim during the 12-month follow-up period, accounting for $11.5 \%$ of the total triptan cost incurred by the health plan for this cohort. For new triptan users, triptan use in the first or second quarter was correlated with triptan use in the entire 12-month follow-up period ( $r=0.187$ and 0.279 , respectively). The mean migrainerelated pharmacy cost per patient during the follow-up was $\$ 871$; however, continuous users had mean costs $(\$ 1,505)$ nearly 3 times the mean costs for new users $(\$ 506, P<0.05)$. The average treatment effect of drug prophylaxis in moderate-to-severe migraine patients was a decrease of $\$ 560$ ( $\$ 514-\$ 607)$ per patient per year in 1998-2001 dollars.

CONCLUSION: High utilizers of migraine therapy can be identified early in treatment. Drug prophylaxis for migraine is cost saving, and an intervention program that increases the use of migraine prophylaxis in potential candidates could be cost beneficial.

KEYWORDS: Migraine, Prophylaxis, Economics, Cost, Managed care

J Manag Care Pharm. 2005;11(2):137-44

Authors

LIDA R. ETEMAD, PharmD, MS, is a researcher, Ingenix, Inc., Eden Prairie, Minnesota (she was a WellPoint/Pharmacia fellow at the University of Southern California, Los Angeles, at the time of this study); WINNIE YANG, PharmD, $B C P S$, is director, Health Informatics and Policy Research, WellPoint Pharmacy Management, West Hills, California; DENISE GLOBE, PhD, was an assistant professor, University of Southern California, Los Angeles, at the time of this study; ARIE BARLEV, PharmD, MS, is senior scientist, Covance, San Diego, California; KATHLEEN A. JOHNSON, PharmD, PhD, is associate professor, departments of Pharmacy and Pharmaceutical Economics and Policy, and vice chair, Department of Pharmacy, University of Southern California, Los Angeles.

CORRESPONDING AUTHOR: Lida R. Etemad, PharmD, MS, Researcher, Ingenix, Inc.,12125 Technology Dr., MN002-0258, Eden Prairie, MN 55344. Tel: (952) 833-7036; Fax: (952) 833-6045; E-mail: lida.etemad@ingenix.com Copyright $\odot$ 2005, Academy of Managed Care Pharmacy. All rights reserved.
$\mathrm{M}$ igraine is a chronic, episodic disorder that interrupts the patient's ability to function and decreases quality of life. It affects $17.6 \%$ of females and $5.7 \%$ of males in the United States, ${ }^{1}$ occurring most commonly between the ages of 25 and $55 .^{2}$ The economic burden of migraine includes both direct and indirect costs. The direct costs are those that are experienced by the patient or the third-party payer resulting from physician office visits, emergency room or urgent care center visits, medications, diagnostic tests, and hospitalizations. Patients and employers also incur indirect costs as a result of missed days of work, decreased productivity while experiencing a migraine, and decreased quality of life.

Annual estimates for the direct costs of migraine care in the United States in 1994 have been estimated at approximately $\$ 1$ billion. $^{3}$ In 1989 and 1990, managed care patients with migraine incurred an average cost of $\$ 145$ per member per month (PMPM) as opposed to $\$ 89$ PMPM for those patients without migraine. ${ }^{4}$ Even though this time period was prior to the introduction of the triptan medications, these patients generated 3 times as many pharmacy claims as the comparison group. A study of the Idaho Medicaid population in 1998 found that migraine patients, on average, incurred $\$ 2,844.67$ in prescription claims per year as compared with $\$ 998.80$ for controls matched on age, sex, and residence. ${ }^{5}$

Utilization of health care services is another method to assess the economic impact of migraine. Joish et al. also found that physician visit, hospital, and outpatient hospital claims were significantly higher in migraine patients. ${ }^{5}$ In 1994, physician office visits accounted for the greatest proportion (60\%) of treatment costs, while prescription medications made up almost all of the remaining costs (30\%).

The severity of attacks varies across migraine patients and can vary even across attacks within one patient. Migraine attacks may range from mild, treatable with simply over-the-counter medications, to so severe that the patient requires a day or more of bed rest. Treatment, therefore, can be complicated and must be individualized. Research analyzing migraine costs by severity level is limited; however, patients with greater severity of migraines show higher rates of consultations. ${ }^{6}$ Severity is generally determined by frequency of headaches, pain intensity, disability, days missed from work, and days of impaired work function. ${ }^{7-9}$

Migraine prophylaxis may be indicated in patients with frequent or severe episodes. It has been suggested that patients experiencing more than 2 attacks per month are candidates 


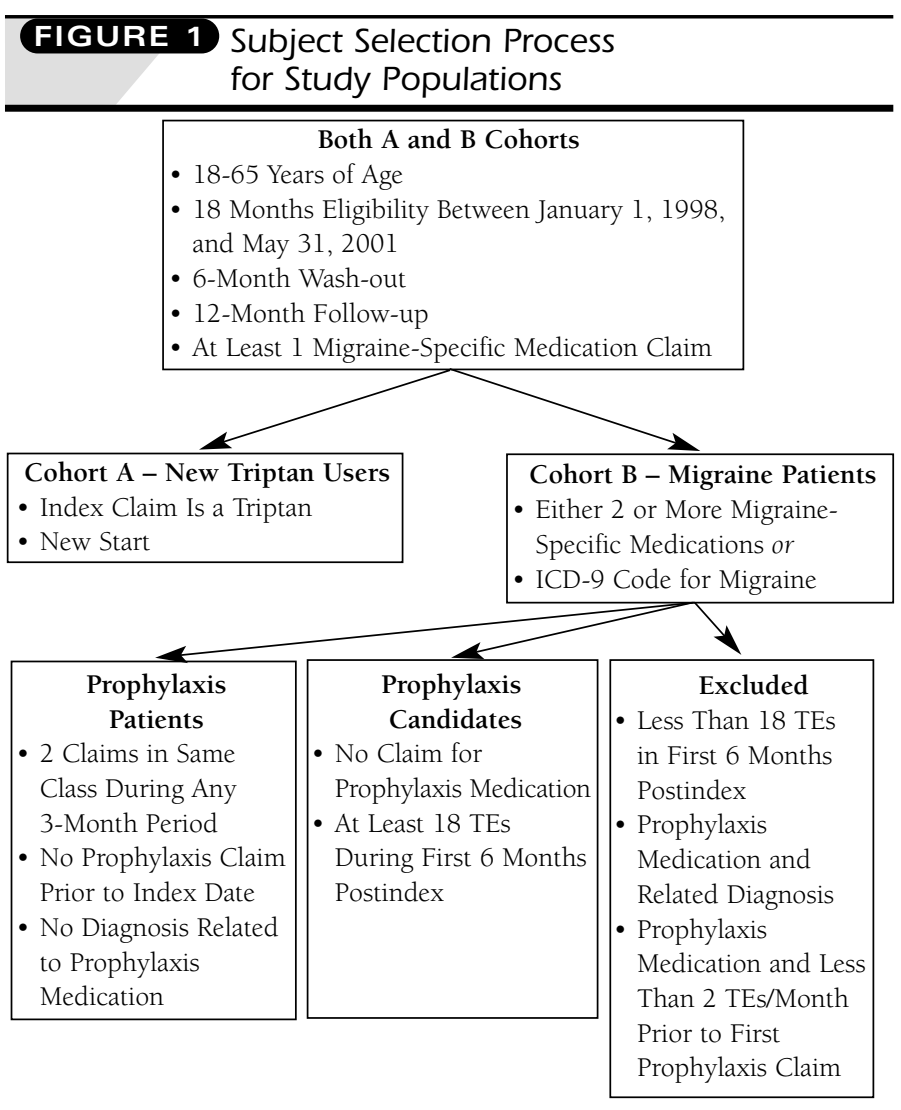

ICD-9=International Classification of Diseases, 9th edition; $T E=$ triptan equivalent.

for prophylaxis. ${ }^{10,11}$ Consensus guidelines for initiation of prophylaxis have not been established. As with acute therapy, the decision to incorporate pharmacologic prophylaxis in a migraineur's regimen is individualized, based on the severity and frequency of attacks and the comorbid conditions of each patient. ${ }^{12}$ Medications used for prophylaxis include beta-blockers, tricyclic antidepressants, valproic acid and derivatives, selective serotonin reuptake inhibitor (SSRI) antidepressants, nonsteroidal anti-inflammatory drugs, and calcium channel blockers. The American Academy of Neurology published migraine treatment guidelines in 2000 that classified the various migraine prophylaxis drugs into 5 categories based on the evidence of efficacy and the incidence of side effects for each drug. Amitriptyline, divalproex, propranolol, and timolol were classified as "Group 1" agents: medications with proven high efficacy and mild-to-moderate adverse events. ${ }^{13}$

Since the introduction of the "triptan" class of medications in the early 1990s, research efforts in migraine have focused on the development of appropriate treatment strategies using this efficacious, yet comparatively expensive, class of medications. Although there has been much research on the cost-effectiveness of sumatriptan therapy, there has been relatively little work done in the area of cost-effectiveness or cost benefit of migraine prophylaxis, presumably because most are now available generically and are relatively inexpensive. A model developed by Steiner in 1995 assesses the cost-effectiveness of migraine prophylaxis but relies heavily on many assumptions. ${ }^{14}$ Adelman et al. developed a model to calculate the minimum number of migraine attacks per month a patient would need to experience for different types of prophylaxis to be cost effective. This method, however, is individualized per patient and would require alteration and population assumptions in order to be applied in a systematic manner in a managed care population. ${ }^{15}$

In addition, there is little research in the area of utilization patterns of triptan users. Characterizing triptan utilization patterns of patients receiving this type of therapy would help to inform the development of disease management programs. Disease management programs may be most efficient when it is possible to identify potentially high utilizers of pharmaceuticals early in drug therapy since these patients may benefit most from drug prophylaxis of migraine. Therefore, information on the feasibility of early identification of high utilizers would augment a disease management intervention.

This study examined the pharmacy and medical utilization and costs of migraine patients in a managed care population and compared health services utilization trends of patients who are treated with prophylaxis with those who might be candidates but do not receive migraine prophylaxis. Triptan use patterns following initiation are evaluated to determine whether potential high utilizers may be identified early in therapy when intervention may be most cost effective. Potential differences in cost among prophylaxis groups was determined to aid in the development of quality improvement initiatives.

\section{Methods}

\section{Study Design}

A retrospective claims analysis was performed utilizing pharmacy, medical, and eligibility databases from a large managed care health plan with more than 2 million members. The health plan used in the study was a mix of health maintenance organization (HMO, 40\%) and preferred provider organization (PPO, 60\%) health plans located in the western United States.

\section{Patient Populations}

Figure 1 illustrates the selection process used to define the patient populations. Commercial enrollees between the ages of 18 and 65 years who had at least 1 migraine-specific prescription claim during the time period of July 1, 1998, to May 31, 2000, were identified. Migraine-specific medications used for the purpose of identifying migraine patients included ergotamine products, isometheptene combination products, methysergide, sumatriptan, zolmitriptan, naratriptan, and rizatriptan. The first migraine-related claim (either pharmacy or medical) was identified as the index claim. Patients who did not have continuous eligibility for the health plan for the 6 months prior to and the 
12 months following the index claim were excluded from the analyses. Two patient cohorts were then identified using the criteria described below.

The first analysis cohort (A) was a subset of patients who were newly started on triptan therapy. This cohort included patients who received a triptan medication as the index pharmacy claim. Patients receiving a migraine-specific medication during the 6 months prior to the index claim were excluded from this cohort. Prescription medication use in the 12 months following the index claim was determined and analyzed in order to characterize migraine-related pharmacy use in new utilizers of triptan medications.

The second analysis cohort (B) represents migraine patients and includes patients who had either (1) an International Classification of Diseases, 9th edition (ICD-9) code for migraine (346.0-346.2, 346.8, and 346.9) and at least 1 migraine-specific medication claim or (2) 2 or more migraine-specific medication claims during the specified time period. Migraine-specific medication claims were identified by the First Data Bank national drug data file definition using Smart-key code 0272 and included ergotamine products, isometheptene combination products, methysergide, sumatriptan, zolmitriptan, naratriptan, and rizatriptan (almotriptan, frovatriptan, and eletriptan were not approved for use in the United States at the time of this study).

\section{Definition of Prophylaxis Subgroup}

Patients in the second analysis cohort (B) were used to identify the subgroups used for the prophylaxis analysis. Patients receiving drug prophylaxis and potential candidates for prophylaxis were identified. The definition for potential candidates for prophylaxis was conservative in order to minimize potential bias. Patients who were potential candidates for prophylaxis were identified based on the suggested criteria that recommends use in patients with greater than 2 migraine attacks per month. ${ }^{12}$ Patients who were determined to be candidates could not have a claim for a medication used for prophylaxis in the 12 months following their index date and were required to possess triptan medication in sufficient quantities to treat more than 18 headaches in the first 6 months following the index migraine-related claim.

Because of the different dosing schedules of the triptan medications, the quantity of triptan medication received was standardized using a proxy unit, the triptan equivalent (TE). Each TE was equal to the maximum quantity recommended to treat 1 headache episode for each triptan medication. Table 1 depicts the quantities for each medication that were considered equivalent to $1 \mathrm{TE}$. Patients possessing greater than $18 \mathrm{TEs}$ in the first 6 months following the index claim were considered potential candidates for migraine prophylaxis medication. The use of these criteria selected only those with frequent migraines (moderate-to-severe migraineurs) for the migraine prophylaxis candidates group.

Patients were considered to be on migraine prophylaxis if

\begin{tabular}{l|c}
\hline \multicolumn{1}{c}{ TABLE 1) } & $\begin{array}{c}\text { Quantity of Triptan Units } \\
\text { Used per Triptan Equivalent }\end{array}$ \\
\hline Medication* $^{*}$ & Quantity per Triptan Equivalent \\
\hline Sumatriptan tablets & 3 tablets \\
\hline Sumatriptan injectable & 2 injections/l kit \\
\hline Sumatriptan nasal spray & 2 doses/1 kit \\
\hline Zolmitriptan tablets & 3 tablets \\
\hline Naratriptan tablets & 2 tablets \\
\hline Rizatriptan tablets & 3 tablets \\
\hline
\end{tabular}

* All doses were considered equivalent in determination of triptan equivalents; therefore, only quantity was used in the calculation. Almotriptan, frovatriptan, and

eletriptan were not approved for use in the United States at the time of this study.

they had at least 2 claims for medications in the same class in any 3-month period during the 12-month follow-up period. Migraine prophylaxis medications included tricyclic antidepressants, SSRI antidepressants, mirtazapine, venlafaxine, phenelzine, beta-blockers, calcium-channel blockers, valproic acid and derivatives, gabapentin, tiagabine, topiramate, and carbamazepine. ${ }^{12}$ Exclusion criteria were applied in order to increase the likelihood that patients were using the medication for migraine prophylaxis versus another nonmigraine indication. Patients who received the prophylaxis medication prior to the migraine index date or who had an ICD-9 code for a disease state related to the prophylaxis medication were dropped from the analysis (i.e., patients receiving a beta-blocker/ calcium channel blocker who had a diagnosis in medical claims for hypertension [ICD-9 code of 401-405, 410, 411, 413, 414] or patients receiving a tricyclic/SSRI who had a claims diagnosis of depression [ICD-9 code of 296.2, 296.3] were excluded).

In order to increase the likelihood that patients in the prophylaxis comparison group experienced migraine headaches in the same frequency as those patients identified as potential prophylaxis patients, an additional exclusion criterion was applied. The number of TEs received during the period from the index date to the date of prophylaxis initiation was calculated. Patients were excluded if they received, on average, fewer than 2 TEs per month during this time period.

\section{Variable Definitions}

Migraine-related medical service utilizations were those associated with an ICD-9 code for migraine (346.0-346.2, 346.8, and 346.9). Costs were calculated as the amount paid by the health plan and did not include patient copays or deductibles. Patient comorbidity was determined by ICD-9 codes obtained from medical claims and was used in the identification of the subset of patients who were using prophylaxis medications.

Patients were defined as having used a migraine-specific medication other than a triptan if they had at least 1 claim for an ergotamine product, isometheptene combination product 


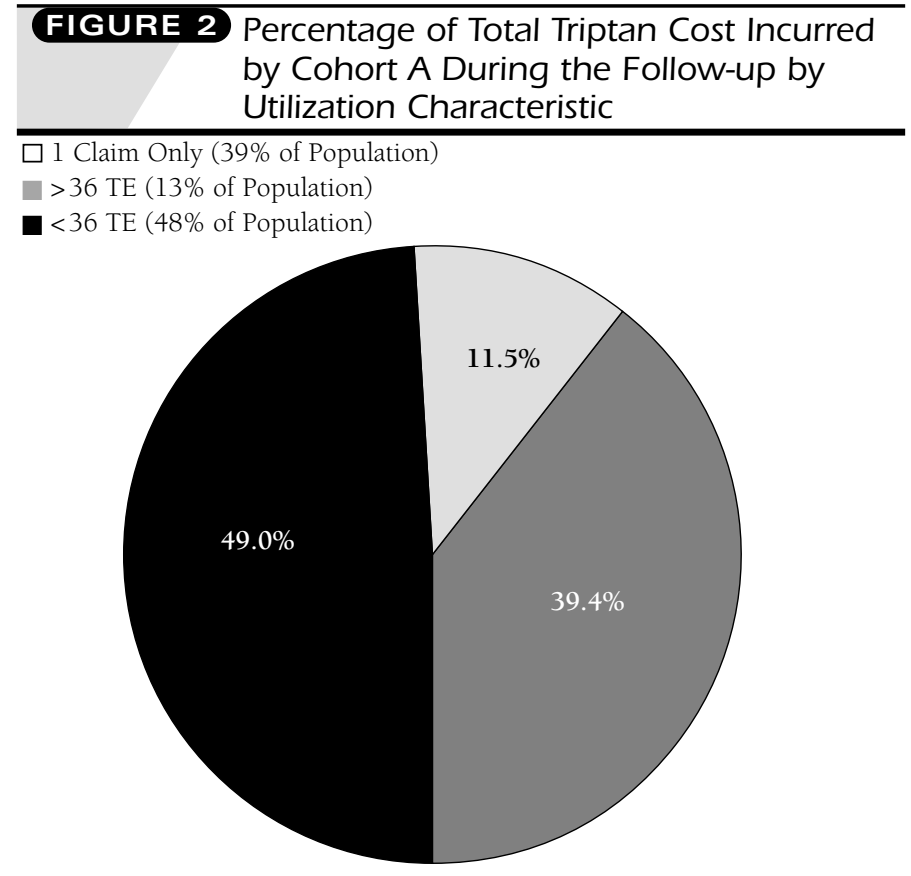

TE= triptan equivalent.

\section{TABLE 2 Prediction of 12-Month Utilization With Utilization During the 1 st and 2nd Quarter Post-Triptan Initiation (Cohort A)}

\begin{tabular}{l|c|c}
\hline Measurement & 1st Quarter Data & 2nd Quarter Data \\
\hline $\begin{array}{l}\text { Correlation of the number of TEs } \\
\text { received in the quarter to the } \\
\text { number of TEs received during } \\
\text { the entire follow-up }\end{array}$ & 0.187 & 0.279 \\
\hline $\begin{array}{l}\text { Odds ratio (95\% CI) of } \\
\text { receiving } \geq 36 \text { TEs* }\end{array}$ & 8.9 & 18.1 \\
\% of patients with $>36$ TEs in \\
the follow-up who had $\geq 9$ TEs \\
during the quarter
\end{tabular}

* Odds ratio for patients with $\geq 9$ TEs (triptan equivalents) during the quarter compared with those with $<9$ TEs).

(and other equivalent products), or methysergide during the 12-month follow-up. The total number of unique migraine medications used was the number of unique medications received during the follow-up period (e.g., a patient with claims for both rizatriptan and sumatriptan during the follow-up would have 2 unique medications).

Patients in the migraine cohort were classified as either newly starting migraine therapy (of any type, not just triptan therapy) or continuous users. New starts were defined as those patients who did not have a migraine-specific pharmacy claim in the 6 months preceding the index claim. Continuous users were defined as patients who had a migraine-specific pharmacy claim in the 6 months preceding the index claim. By definition, continuous users had an index date between July 1, 1998, and December 31, 1998; however, the index date for new starts ranged from July 1, 1998, to May 31, 2000.

\section{Analyses}

Triptan utilization statistics were calculated for those patients identified in the new triptan users cohort. The number of patients who received only 1 prescription for a triptan medication was calculated. The total cost of migraine-specific medications received by the cohort in the 12-month follow-up was determined. The quantity of TE received in the first quarter, second quarter, and 12 months following the index triptan claim was determined. The correlation between consumption of triptan medication early in treatment (in the first and second quarters) and the entire follow-up was calculated.

Descriptive demographic and utilization statistics were compared between the prophylaxis and prophylaxis candidate subgroups using $t$ tests and chi-square tests where appropriate. All analyses were conducted using SAS version 8.0 (SAS Institute, 2001).

The average treatment of migraine drug prophylaxis was also determined using a method that incorporated ordinary least squares (OLS) regression analysis. Since cost data had no upper bound and the distribution was therefore skewed to the right, the log of the cost was used as the outcome variable. Taking the $\log$ of the cost resulted in a more normally distributed variable. OLS regression analysis was then used to determine the association of drug prophylaxis use with migraine-related health care costs in the follow-up period for the subset of patients identified as either using prophylaxis or being candidates for prophylaxis. The following variables were used in the model: use of drug prophylaxis, sex, age, continuous user, use of a nontriptan migraine-specific medication, number of unique migrainespecific medications used, and concurrent use of 2 or more unique triptans.

Using the model estimated by the OLS regression, a treatment effect for each patient was calculated by retransforming the logged equation (including the sample variance as a correction term) and predicting total costs for each patient utilizing his or her individual parameters. The treatment effect (TxE) for each patient was calculated as the total predicted migraine cost when the variable indicating prophylaxis use was set equal to 1 minus the total migraine cost predicted when it was set equal to 0 . The average treatment effect (ATE) is then the mean of the treatment effects for the entire population:

$$
\mathrm{ATE}=\Sigma(\mathrm{TxE}) / \mathrm{h}
$$

\section{Results}

\section{New Triptan Users}

A total of 5,294 patients were identified as having a new triptan prescription claim during the study time period (Cohort A). 
Costs and Utilization of Triptan Users Who Receive Drug Prophylaxis for

Migraine Versus Triptan Users Who Do Not Receive Drug Prophylaxis

TABLE 3 Demographic and Utilization Characteristics of Cohort B, New Starts, and Continuous Users

\begin{tabular}{|c|c|c|c|c|c|c|}
\hline & $\begin{array}{c}\text { Entire } \\
\text { Population }\end{array}$ & (SD) & New Starts & (SD) & $\begin{array}{c}\text { Continuous } \\
\text { Users }\end{array}$ & (SD) \\
\hline \multirow[t]{2}{*}{ Number } & 8,488 & & 5,382 & & 3,106 & \\
\hline & & & $63.4 \%$ & & $36.6 \%$ & \\
\hline Age & 42.9 & $(9.9)$ & 41.31 & $(10.1)$ & $45.55^{*}$ & $(9.0)$ \\
\hline$\%$ female & $83.6 \%$ & & $82.3 \%$ & & $85.8 \% *$ & \\
\hline Number of standardized triptan equivalents received (median) & 16 & & 11 & & $30 *$ & \\
\hline Total number of migraine ER visits per 100 patients per year & 7.35 & $(72.4)$ & 6.15 & $(41.5)$ & 9.43 & $(106.4)$ \\
\hline Total number of migraine hospitalizations per 100 patients per year & 2.00 & $(32.0)$ & 1.88 & $(26.3)$ & 2.22 & $(39.9)$ \\
\hline Total number of migraine office/outpatient visits per 100 patients per year & 100.71 & $(316.6)$ & 95.75 & $(238.0)$ & 109.30 & $(419.2)$ \\
\hline Total Rx migraine cost (PPPY) & $\$ 871.32$ & $(1,392.47)$ & $\$ 505.83$ & $(698.82)$ & $\$ 1,505.32 *$ & $(1,959.87)$ \\
\hline Total cost of migraine ER visits (PPPY) & $\$ 5.35$ & $(56.96)$ & $\$ 4.57$ & $(39.68)$ & 6.69 & $(78.34)$ \\
\hline Total cost of migraine hospitalizations (PPPY) & $\$ 28.13$ & $(531.0)$ & $\$ 26.65$ & $(429.41)$ & 30.71 & $(671.86)$ \\
\hline Total cost of migraine office/outpatient visits (PPPY) & $\$ 82.16$ & $(300.6)$ & $\$ 81.03$ & $(258.65)$ & 84.12 & $(362.00)$ \\
\hline Total cost of migraine medical utilization (PPPY) & $\$ 117.98$ & $(688.26)$ & $\$ 115.50$ & $(550.00)$ & 122.28 & $(877.80)$ \\
\hline Total migraine costs (PPPY) & $\$ 989.00$ & $(1,583.89)$ & $\$ 620.93$ & $(887.52)$ & $1,627.60^{*}$ & $(2,202.10)$ \\
\hline
\end{tabular}

* Significantly different between new triptan starts and continuous triptan users $(P<0.05)$. ER = emergency room; $R x=$ prescription; PPPY = per patient per year for $1998-2001$ costs.

Thirty-nine percent of patients received only 1 triptan claim during the 12-month follow-up period, accounting for $11.5 \%$ of the total triptan cost incurred by the health plan for this cohort (Figure 2).

The number of patients exceeding 36 TEs (the quantity sufficient to treat greater than 2 migraines per month) during the follow-up year was also calculated. Thirteen percent of new triptan users had greater than 36 TEs and accounted for 39\% of the total triptan cost incurred by the cohort.

The amount of TEs received in either of the first or second quarters was correlated with the total number of TEs received in the 12-month follow-up (Table 2). Patients who received more than 9 TEs in the first 3 months following their index date were 8.9 (95\% CI, 7.0-11.4) times more likely to exceed 36 TEs in the 12-month follow-up than those who received fewer than 9. Combining the first- and second-quarter data resulted in an increased correlation coefficient (0.284) as compared with either quarter alone; however, using data from either quarter correctly identified more than $26 \%$ of patients who exceeded 36 TEs in the 12-month period.

\section{Migraine Patients}

\section{Demographic and Descriptive Statistics}

A total of 8,488 patients were identified as meeting the inclusion criteria. Descriptive statistics for the overall study cohort are given in Table 3. The overall total migraine cost to the health plan per patient during the 1-year follow-up period was $\$ 989.00$ per person per year (PPPY), with pharmacy costs accounting for the majority of the total costs (88.1\%).
Continuous users were significantly older than the new users and had significantly greater pharmacy utilization and, consequently, greater total costs.

\section{Prophylaxis Versus Prophylaxis Candidates}

Table 4 describes the utilization characteristics of the subset of patients included in the prophylaxis analysis. Patients receiving drug prophylaxis were younger than those not receiving prophylaxis. Although the total costs of migraine medications were significantly lower for those receiving drug prophylaxis, those on prophylaxis had more migraine-related hospitalizations and outpatient office visits (which resulted in higher overall medical utilization cost) compared with those not receiving prophylaxis. These differences remained statistically and significantly different when the Wilcoxon rank sum test was performed to test for differences in non-normally distributed variables.

The results of the OLS regression with independent variables included are shown in Table 5. The coefficient on the treatment variable (prophylaxis use) predicts approximately a $21 \%$ decrease in total migraine cost for patients who use drug prophylaxis. Retransforming the equation, the predicted average treatment effect of migraine prophylaxis across the population was a $\$ 559.71$ reduction in total migraine cost (95\% CI, \$514.28-\$607.26) PPPY (in 1998-2001 dollars).

\section{Discussion}

The patients in this population resembled those in earlier studies, with the majority being female and between the ages of 


\begin{tabular}{|c|c|c|c|c|c|}
\hline & Prophylaxis & $(\mathrm{SD})$ & No Prophylaxis & $(\mathrm{SD})$ & $P$ Value \\
\hline umber (total $=1,124)$ & 286 & & 838 & & \\
\hline & $25.4 \%$ & & $74.6 \%$ & & \\
\hline ge & 42.39 & $(10.1)$ & 45.13 & (8.8) & $<0.0001$ \\
\hline female & $82.9 \%$ & & $80.30 \%$ & & 0.3832 \\
\hline ledian number of standardized triptan equivalents received & 36 & & 45 & & 0.0335 \\
\hline tal number of migraine ER visits per 100 patients per year & 23.43 & $(267.2)$ & 4.06 & $(30.7)$ & 0.2223 \\
\hline tal number of migraine hospitalizations per 100 patients per year & 5.59 & $(38.0)$ & 0.48 & $(6.9)$ & 0.0242 \\
\hline tal number of migraine office/outpatient visits per 100 patients per year & 185.66 & $(647.9)$ & 67.66 & $(188.4)$ & 0.0026 \\
\hline tal Rx migraine cost (PPPY) & $\$ 1,691.84$ & $(1,735.87)$ & $\$ 2,011.71$ & $(1,738.70)$ & 0.0073 \\
\hline tal cost of migraine ER visits (PPPY) & $\$ 17.14$ & $(194.06)$ & $\$ 2.23$ & $(19.70)$ & 0.1957 \\
\hline tal cost of migraine hospitalizations (PPPY) & $\$ 39.23$ & $(383.79)$ & $\$ 8.15$ & $(210.58)$ & 0.1957 \\
\hline tal cost of migraine office/outpatient visits (PPPY) & $\$ 163.81$ & $(714.45)$ & $\$ 54.06$ & $(204.11)$ & 0.0109 \\
\hline tal cost of migraine medical utilization (PPPY) & $\$ 219.74$ & (1193.56) & $\$ 65.18$ & (308.09) & 0.0312 \\
\hline tal migraine costs (PPPY) & $\$ 1,911.57$ & $(2371.61)$ & $\$ 2,076.89$ & $(1,767.00)$ & 0.2804 \\
\hline
\end{tabular}

* Potential candidates received at least 18 triptan equivalents in the 6-month postindex period and received no migraine prophylaxis medication. $E R=$ emergency room; $R x=$ prescription; $P P P Y=$ per patient per year for 1998-2001 costs.

25 and 55 years. ${ }^{1,2}$ Although the present study had a higher percentage of females than previously reported, ${ }^{1}$ the previous study was a population-based study whereas the present study occurs in a treated cohort. Since women are known to seek medical treatment more often than men, this finding is not surprising and probably reflects real-world health plan treatment populations.

The migraine-related cost incurred by this population is consistent with other studies. In 1999, migraineurs in a managed care population in western Pennsylvania had an average of $\$ 97.85$ per PPPM in migraine-related costs prior to a triptan quantity limit intervention. This is similar to the $\$ 989$ in migrainerelated health care costs PPPY (\$82.42 PPPM) in our study.

Unlike the study conducted by Hu et al., the pharmacy costs in our study dominated the medical costs, accounting for $88.1 \%$ of the overall cost. Due to the definition of a migrainerelated medical visit (ICD-9 code for migraine in the first 2 diagnosis fields), it is possible that the true medical costs may have been underestimated due to probable undercoding of medical service claims for migraine. However, with a proportion this high, it is reasonable to conclude that pharmacy costs represent a majority of the cost of migraine therapy and that the potential under-estimate of outpatient costs would not significantly bias the total cost estimates.

Continuous triptan users utilized considerably more migraine pharmacy services than the patients newly initiating therapy. It could be postulated that this group of patients is more refractory to migraine medications. Further characterization of utilization patterns in this subset could lead to greater insight into the prevalence of dose and quantity escalation and the possibility of rebound headaches.

Predicting which patients will be high utilizers early in triptan therapy appears to be feasible. The amount of triptan used in the first 6 months following triptan initiation is correlated with the amount used in the entire 12-month period. Identification and implementation of quality assurance programs in potential high users early in treatment can be beneficial to managed care plans in reducing migraine cost burden and to patients in modifying the burden of migraines.

The majority of the migraine patients identified by medical claims with a migraine ICD-9 were new starts (78.8\%). For new-start patients with a medical claim as the index claim, 17\% did not receive a triptan medication in the follow-up period and $35.6 \%$ received only 1 triptan claim. Patients who received only 1 triptan claim may have been prescribed the medication to aid in diagnosis of migraine versus tension headaches (since tension headaches do not respond to triptan medications). Due to the high cost of triptan medications, however, this is not a preferred method of differential diagnosis of headaches. Since this represents a substantial portion (35.6\%) of the patients identified by index medical claims, prescribers with multiple patients in this group may benefit from an intervention program that outlines the International Headache Society criteria for migraine diagnosis.

Overall, patients receiving drug prophylaxis had lower migraine-related costs than those using acute treatment alone. This information is useful to any health care payer seeking to 
reduce migraine-related costs. Any intervention to increase the use of migraine prophylaxis in appropriate patients that costs less than $\$ 559.71$ PPPY in which drug prophylaxis is initiated has the potential to be cost saving.

Nearly $10 \%$ of the total population and $8 \%$ of the new triptan users fit the criteria for inclusion in the prophylaxis candidate subgroup. Since it is possible to identify potential prophylaxis candidates during the first 6 months following initiation of therapy, a migraine intervention program is feasible in the early stages of treatment.

Patients for this study were identified over a period of 23 months. During this time period, migraine therapy improvement initiatives in the form of physician letters to increase the use of migraine prophylaxis were undertaken by the health plan. While these types of initiatives may normally lead to possible introduction of bias, the majority of patients for this study (75\%) were identified in the first 6 months of the study, alleviating this potential issue. In addition, the month of subject identification was also entered into the multivariable regression and was found to be nonsignificant, indicating that any temporal treatment pattern changes were not significant predictors of cost.

This study examined the potential economic impact of drug prophylaxis in a managed care migraine population and the feasibility of intervening early in therapy. Further study to characterize patients who receive migraine drug prophylaxis may provide additional information to the design of a clinical intervention program.

\section{Study Limitations}

This study only takes into account direct costs to the health plan and does not include any higher or lower costs due to beneficial effects from reduced disability or detrimental side effects of drug prophylaxis for migraine. Patients who require bed rest while experiencing a migraine attack may feel that the reduced direct costs understate the overall benefit since the reduction in number and severity of migraine episodes allows the patients to be more productive and have a higher quality of life. Others who experience less-severe migraine episodes may believe that the side effects of the medications for prophylaxis are troublesome and offset any reduction in number of migraine episodes. Further work to incorporate these aspects into a costeffectiveness study would be beneficial from a societal standpoint.

This study has the limitations associated with any retrospective claims database analysis. Due to miscoding or absence of coding, some medical claims may be inappropriately classified as non-migraine-related when migraine care was actually provided. Patients may have an office visit for a routine check-up and also receive a prescription for a triptan medication without the visit reflecting a diagnosis code for migraine. In addition, patients may have been receiving a medication for prophylaxis for another indication without a claims diagnosis of that indication appearing during the study period (e.g., SSRIs for

\section{TABLE 5 OLS Regression Analysis of Log Migraine- Related Costs on Prophylaxis Use}

\begin{tabular}{l|c|c}
\hline Variable & $\beta$ Coefficient & $P$ Value \\
\hline Intercept & 6.88 & $<0.0001$ \\
\hline Drug prophylaxis & -0.21 & $<0.0001$ \\
\hline Continuous user & 0.36 & $<0.0001$ \\
\hline Concurrent triptan use & 0.29 & $<0.0001$ \\
\hline Age & 0.01 & 0.0048 \\
\hline Female & -0.06 & 0.2501 \\
\hline Use of nontriptan & -0.11 & 0.1809 \\
\hline F statistic & 26.01 & $<0.0001$ \\
\hline Adjusted $R^{2}$ & & 0.1179 \\
\hline OLS=ordinary least squares. & & \\
\hline
\end{tabular}

depression). This would lead to the inclusion of patients who were not primarily receiving their drug prophylaxis for migraine therapy. However, while we excluded patients in which competing medical indications were present in the claims in order to be methodologically conservative, patients receiving medications with a prophylaxis effect would receive this benefit of the medication regardless of the indication and this should not bias the treatment-effect results.

While claims data allow for the determination of the medications received by patients, they do not allow for determination of how medications were used. This is the principal limitation of claims data analysis, particularly when examining medications that are taken as needed rather than in a prescribed regimen. Additionally, use of over-the-counter medications was not captured. This study included patients who received sufficient quantities of triptans to treat at least 2 migraine attacks per month for the specified time period. While it is possible that patients used greater than 1 triptan equivalent to treat 1 migraine episode, did not use the entire quantity received during the time period outlined, or used medications other than triptans to treat migraine attacks, it is unlikely that these types of patterns differed between the treatment groups studied.

Patient comorbidities were not controlled for in this analysis. Due to the potential of miscoding, in many cases, it is important to adjust for other disease states that may influence health services cost. During model development, we attempted to include dichotomous variables that indicated the presence of disease states that are common comorbidities of migraine headache (e.g., depression). These variables proved to be nonsignificant. This result is most likely due to the fact that only migraine-specific cost was included in the outcome and that this cost was highly driven by migraine-specific drug use. Had total health services cost been utilized as the outcome of interest, controlling for comorbidities might have provided additional information (i.e., resulted in significant coefficients). 
Medical criteria for prophylaxis initiation have not been uniformly developed and are based on not only migraine frequency but disability as well. Due to the nature of a claims database, qualitative effects of the migraine episodes could not be assessed; therefore, the quantity of triptan medication received was used as a proxy to determine if drug prophylaxis was indicated. This may have inappropriately categorized patients with frequent but less-severe migraines and those with infrequent but extremely debilitating migraines. Although we attempted to control for any variation in migraine severity and frequency by the subgroup definition, it is possible that variations in these factors may not have been fully accounted for.

Since these are real-world data not derived from a randomized, placebo-controlled trial, it is possible that selection bias may have occurred. Physicians may have based treatment decisions on clinical factors that could not be captured in the claims dataset. By implementing strict inclusion criteria, we attempted to control for this. Patients included in the subgroup analysis were similar with respect to triptan consumption prior to the initiation of prophylaxis therapy. To control for selection bias, we conducted 2 additional analyses using a model that included a propensity score ${ }^{17}$ and a model based on matched cases. However, the results remained the same, supporting the methodology used and the fact that appropriate inclusion and exclusion criteria were utilized to identify patients that were comparable. This study examined costs and utilization in a managed care health plan that was a mix of HMO and PPO models, and the results may not apply to other health plans.

Finally, this model explains a small proportion (12\%) of variation in migraine-related medical cost between migraine patients. There are other factors that influence overall cost in addition to those we were able to determine from a claims dataset.

\section{Conclusion}

Migraine is a costly disorder for health plans. Identification of utilization characteristics is useful in developing disease management programs aimed at increasing quality patient care and decreasing overall costs. Since there is a strong correlation between use in the first 6 months following triptan initiation and the entire first year of follow-up, it is possible to identify high triptan utilizers early in treatment. Continuing triptan patients are much more costly than new starts. It is therefore potentially valuable to a health plan to identify patients who would benefit from an intervention program early following triptan therapy initiation. Patients receiving greater than 18 TEs in a 6-month period may benefit from the use of migraine drug prophylaxis. Migraine drug prophylaxis is cost-saving ( $\$ 559.71$ per patient in 1998-2001 dollars), and an intervention program that increases the use of migraine drug prophylaxis in potential candidates could be cost beneficial.

\section{DISCLOSURES}

This study was funded through the support of a fellowship for graduate study and data extraction by Pharmacia Corporation, now part of Pfizer, Inc.; funding was obtained by author Kathleen A. Johnson. Authors Lida R. Etemad and Arie Barlev were Pharmacoeconomic Research fellows sponsored by Pfizer (formerly Pharmacia) and WellPoint, Inc., at the University of Southern California, Los Angeles, at the time of this study. Johnson discloses that she has served as a consultant for WellPoint and BMS Managed Care Information and has received numerous research grants from pharmaceutical, health care, and educational organizations. Authors Winnie Yang and Denise Globe disclose no potential bias or conflict of interest relating to this article.

Etemad served as principal author of the study. Study concept and design were contributed by Etemad, Yang, and Johnson. Analysis and interpretation of data were contributed by Etemad, Yang, and Barlev. Drafting of the manuscript was primarily the work of Etemad, and its critical revision was the work of Globe and Johnson. Statistical expertise was contributed by Etemad and Barlev.

\section{REFERENCES}

1. Stewart WF, Lipton RB, Celentano DD, Reed ML. Prevalence of migraine headache in the United States. JAMA. 1992;267(1):64-69.

2. Lipton RB, Stewart WF. The epidemiology of migraine. Eur Neurol.1994; 34(suppl 2):6-11.

3. Hu XH, Markson LE, Lipton RB, Stewart WF, Berger ML. Burden of migraine in the United States. Arch Intern Med. 1999;159:813-18.

4. Clouse JC, Osterhaus JT. Health care resource use and costs associated with migraine in a managed health care setting. Ann Pharmacother. 1994;28:659-64. 5. Joish VN, Cady PS, Shaw JW. Health care utilization by migraine patients: a 1998 Medicaid population study. Clin Ther. 2000;22:1346-56.

6. Lipton RB, Stewart WF, Simon D. Medical consultation for migraine: results from the American Migraine Study. Headache. 1998;39(2):87-96.

7. Stewart WF, Shechter A, Lipton RB. Migraine heterogeneity. Disability, pain intensity, and attack frequency and duration. Neurology. 1994:44(6 suppl 4): S24-S39.

8. Stewart WF, Lipton RB, Simon D, Von Korff M, Liberman J. Reliability of an illness severity measure for headache in a population sample of migraine sufferers. Cephalalgia. 1998;18(1):44-51.

9. Stewart WF, Lipton RB, Dowson AJ, Sawyer JM. Development and testing of the Migraine Disability Assessment (Midas) Questionnaire to assess headacherelated disability. Neurology. 2001;56(6 suppl 1):S20-S28.

10. Lance JW. Preventive treatment in migraine. In: Goadsby PJ, Silberstein SD, eds. Blue Books of Practical Neurology: Headache. London: ButterworthHeinemann; 1997:131.

11. Tfelt-Hansen P, Welch KMA. General principles of pharmacologic treatment In: Oleson J, Tfelt-Hansen P, Welch KMA, eds. The Headaches. New York, NY: Raven Press; 1993:299-303.

12. Ramadan NM, Silberstein SD, Freitag FG, Gilbert TT, Frishberg BM. Evidence-based guidelines for migraine headache in the primary care setting: pharmacological management for prevention of migraine. Available at: http://aan.com. Accessed September 25, 2001.

13. Silberstein SD, for the U.S. Headache Consortium. Practice parameter: evidence-based guidelines for migraine headache (an evidence-based review) Report of the Quality Standards Subcommittee of the American Academy of Neurology. Available at: http://www.neurology.org/cgi/reprint/55/6/754.pdf. Accessed December 6, 2004.

14. Steiner TJ. Long-term cost-benefit assessment of anti-migraine drugs. Cephalalgia. 1995;15(suppl 15):37-40.

15. Adelman JU, Frod A, Von Seggern RL, Mannix LK, Rapoport AM. Migraine preventive medications: a reappraisal. Cephalalgia. 1998;18:605-11. 16. Culley EJ and Wanovich RT. Medical and pharmacy cost and utilization outcomes of a quantity limit on the 5-HTl agonists (triptans) by a managed care organization. J Manag Care Pharm. 2001;7(6):468-75.

17. D'Agostino RB. Tutorial in biostatistics propensity score methods for bias reduction in the comparison of a treatment to a non-randomized control group. Stat Med. 1998;17:2265-81. 